\title{
Policymakers' perceptions of oral pre-exposure prophylaxis introduction in Tanzania: Findings from implementation science research
}

Population Council

Follow this and additional works at: https://knowledgecommons.popcouncil.org/departments_sbsr-hiv

Part of the Demography, Population, and Ecology Commons, Family, Life Course, and Society Commons, International Public Health Commons, and the Medicine and Health Commons How does access to this work benefit you? Let us know!

\section{Recommended Citation}

Population Council. 2017. "Policymakers' perceptions of oral pre-exposure prophylaxis introduction in Tanzania: Findings from implementation science research," DREAMS Tanzania Results Brief 6. Washington, DC: Population Council. 
Policymakers and other key stakeholders in Tanzania are currently considering oral pre-exposure prophylaxis (PrEP), as an HIV prevention option for adolescent girls and young women (AGYW). PrEP, antiretroviral medication taken by HIV-uninfected persons to prevent HIV, is recommended by the World Health Organization as a prevention option for people at substantial risk for HIV. ${ }^{1}$ Adolescent girls and young women in Tanzania are particularly vulnerable to HIV. HIV prevalence is 1.3 percent among adolescent girls aged $15-19$ years and 4.4 percent among young women aged 20-24. ${ }^{2}$ If AGYW can access, use, and adhere to PrEP as part of a comprehensive HIV prevention package, their acquisition of HIV might be substantially lowered.

However, there is limited evidence from Tanzania to inform decision-making on whether and how to introduce PrEP to this population. To help fill this evidence gap, the Population Council in collaboration with the National AIDS Control Program (NACP) and CSK Research Solutions Limited conducted implementation science research in one urban district (Dar es Salaam) and one rural district (Mbeya) in Tanzania to explore policymakers' and key stakeholders' perceptions and attitudes toward the introduction of PrEP to different groups, including AGYW, as well as to identify key issues that must be considered prior to its introduction.

\section{METHODS}

Key informant interviews (KIIs) were conducted with 21 policymakers and key stakeholders. Respondents included representatives from the Ministry of Health, Community Development, Gender, Elderly and Children (MoHCDGEC), specifically National AIDS Control Program (NACP) personnel from the national, regional, district, and community level; regional and council medical officers; religious leaders; and representatives from civil society organizations (CSOs) offering HIV prevention services to AGYW in the two study districts. Study script that included questions about financing PrEP, PrEP's place in HIV prevention strategies, and opportunities for and barriers to PrEP introduction. The study was approved by the National Research Ethics Committee of Tanzania and Population Council Institutional review board (New York, USA). 


$\begin{array}{ccc}\begin{array}{c}\text { DAR ES SALAAM } \\ (n=11)\end{array} & \begin{array}{r}\text { MBEYA } \\ (n=10)\end{array} \\ 5 & \text { Sex } & 7 \\ 6 & \text { Male } & 3 \\ 8 & \text { Female } & \\ 3 & \text { Organization } & 7 \\ 0 & \text { Government } & 1 \\ & \text { CSO } & 2\end{array}$

\section{RESULTS}

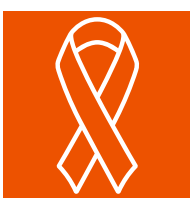

\section{PrEP introduction is viewed as an opportunity to achieve Tanzania's HIV testing targets and to reduce HIV incidence among AGYW.}

Achieving the first 90: The first 90 of UNAIDS 90-9090 targets, refers to the goal of getting 90 percent of all people living with HIV to know their HIV status by $2020 .{ }^{3}$ Because one of the pre-requisites for an individual to take PrEP is to be tested for HIV and test HIV-negative, policymakers indicated that the demand for PrEP could increase the number of people who test for HIV. Therefore, PrEP could help Tanzania get closer to achieving the first 90 target.

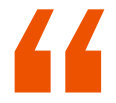

Firstly, we need more people to get tested so it [referring to PrEP] will help us learn the HIV status of our people, so it will increase our coverage because we haven't yet reached the UN target".

-Dar es salaam

HIV incidence reduction among AGYW: Policymakers concurred that introduction of PrEP in Tanzania would contribute positively to the country's efforts toward reducing new HIV infections among AGYW who are at high risk for HIV. They identified specific sub-populations of AGYW as ideal beneficiaries of PrEP, including those in discordant relationships, female sex workers, and those who inject drugs. Policymakers also noted that PrEP would serve to prevent new HIV infections among key populations, including men who have sex with men, prisoners, and drivers who travel long distances.

\begin{abstract}
4 I think mainly will be the youth who have not yet started practicing sexual intercourse so if we go ahead they will benefit much....those from 15-24 years. Other groups include drug addicts who are currently not HIV infected, and drivers who travel long distances, but everyone who is in risk environment can benefit from PrEP."
\end{abstract}

-Mbeya

Some policymakers reported that PrEP would be a good HIV prevention option among individuals who dislike condoms. They explained that many individuals, including those in discordant relationships, do not use condoms for various reasons (e.g., perceived reduction in sexual pleasure) despite the risk of HIV.

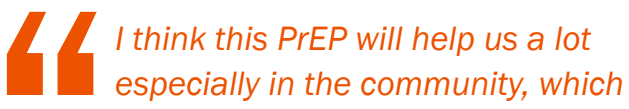
even when we educate them on ways to protect themselves they don't understand, like using a condom, they say they don't feel like using it, so, PrEP might help them".

-Mbeya

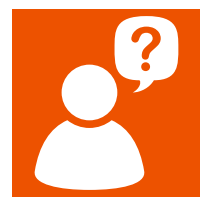

Key concerns about PrEP centered on financing, its potential burden on the health system, appropriate service delivery points, PrEP adherence, and behavioral

\section{disinhibition by users.}

Financing: Policymakers were concerned about the government's ability to afford PrEP. They reasoned that the Tanzanian government could not afford to pay for PrEP, as the country is currently dependent on funding 
support from development partners for the country's HIV program. Additionally, they explained that the health system already has insufficient resources, given the recent reduced support for the Tanzanian basket fund (a mechanism funded by several development agencies that pool un-earmarked resources to support the implementation of the health sector strategic plans) and the need to implement the new HIV policy of "test \& start." Therefore, they expressed concerns about who would fund PrEP and for what period of time.

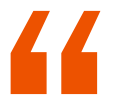

You know the support from global

fund has gone down. There are some policies like test and start which cannot be achieved because the basket fund has much demand and we are not able to provide these medications [referring to ARVs]"

-Dar es Salaam

Burden on the health system: Several policymakers were concerned about the effect PrEP introduction would have on an already over-stretched health staff. Assuming that PrEP might result in an increased number of individuals seeking HIV testing and needing continuous follow-up, policymakers envisioned an increased workload which existing staff might not be able to adequately manage. Other policymakers, however, did not think that client load would be a problem. They believed that PrEP introduction would lead to fewer new HIV infections and hence fewer care and treatment (CTC) clients.

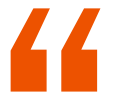

The effect will be there because of the human resources. Because, we don't have enough of them and we are putting this as an additional service"

-Mbeya

All policymakers urged that PrEP be carefully introduced in Tanzania with a special emphasis on health system preparedness to accommodate this new HIV prevention strategy and community awareness building to create demand among potential users. They noted there was a need for training health staff to manage PrEP clients. In addition, they expressed a strong need for locallygenerated evidence around acceptability of the drug (both among potential users and the community at large) as well as adherence to the medication by clients.
46 Making enough preparations in terms of human resources is important. Not only that they should be given the capability through sufficient training but also enough resources should be present in terms of testing equipment and thirdly preparing the community in terms of education. At the end of the day we will see how it goes."

-Mbeya

4 Studies have to be done so as we could make decisions at policy level...Let's do these studies from the Tanzanian perspective including training and ordering and storage and how should we modify our facilities and what does it cost to have PrEP in a facility then you'll be able to convince anyone to set aside funds for PrEP."

-Dar es Salaam

Service delivery points: Some policymakers cautioned about introducing PrEP in current HIV CTCs because of the stigma associated with these centers. They explained that CTCs are known as places where people living with HIV access services and therefore, potential PrEP beneficiaries might shun these locations due to fear they would be judged as having HIV. Hence, they recommended that PrEP be offered in HIV testing service centers as well as by community- based providers and by CSOs. Some policymakers, however, thought PrEP could be embedded in CTCs because the staff is already trained to provide ARV services.

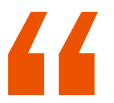
No, it should not be given out in a CTC because it [CTC] is a referral for those who are positive. It should be in testing and counselling centers."

-Dar es Salaam

Fears about adherence and behavioral disinhibition: Policymakers explained that adherence remains a challenge for people living with HIV, whose lives depend on the medication. Therefore, they questioned whether individuals, especially AGYW, who are not yet sick can adhere to PrEP. They were also concerned that individuals would not take the drug as prescribed; instead choosing to take it only prior to or immediately after having unprotected sex. 
46 Unfortunately, I have a lot of doubts... going back to the history of ARVs, adherence among adolescent girls is very poor, even though these are individuals who are already HIV positive and they know that their survival depends on taking ARVs. Now, for a person who's not even HIV positive to start taking the medicine brings in a lot of uncertainties"

-Dar es Salaam

Some policymakers raised concerns regarding the possibility of an increase in risky sexual behaviors because of PrEP use. They noted that many people have engaged in safer sexual practices due to fear of HIV. With diminished fears, individuals, especially adolescents, might engage in high-risk behaviors.

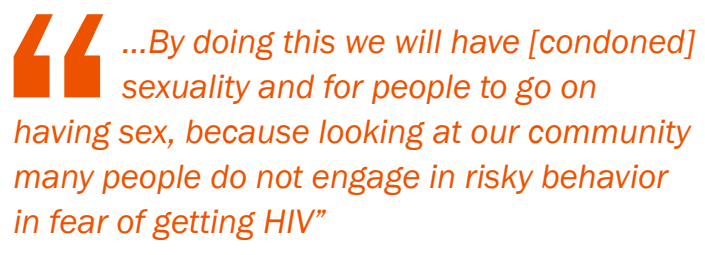

-Mbeya

4 ... For a young girl, it is very easy to decide because I am using medication, I will go and have many affairs but an adult will judge what do I gain from those relationships."

-Dar es Salaam

\section{RECOMMENDATIONS}

- Discuss financing options with Ministry of Finance and Planning and other international stakeholders.

- Conduct cost modeling to see potential cost and benefits of PrEP introduction and costing models to assess how PrEP could be funded.

- Prepare the healthcare system for PrEP introduction by strengthening health providers' capacity to deliver PrEP.

- Consider partnering with community-based providers and organizations to integrate PrEP into HIV testing services to minimize the burden on the government healthcare system and reduce potential stigma.

- Ensure that the community is educated and sensitized about PrEP to minimize potential stigma.

- Continue to support research to generate local evidence around PrEP acceptability and adherence among beneficiaries.

\section{REFERENCES}

${ }^{1}$ Tanzania Commission for AIDS (TACAIDS) et al. 2013. Tanzania HIV/AIDS and Malaria Indicator Survey 2011-12. Dar es Salaam, Tanzania:TACAIDS, ZAC, NBS, OCGS, and ICF International.

${ }^{2}$ WHO. 2015. "Guideline on when to start antiretroviral therapy and on pre-exposure prophylaxis for HIV." Geneva, Switzerland: WHO.

3UNAIDS. 2014. "90-90-90-An ambitious treatment target to help end the AIDS epidemic." Geneva: UNAIDS. unaids.org/sites/default/files/ media_asset/90-90-90_en.pdf
For more information, contact Nanlesta Pilgrim (npilgrim@ popcouncil.org), Lou Apicella (lapicella@popcouncil.org), or Catherine Kahabuka (ckahabuka@cskresearch.com).
POPULATION COUNCIL

Ideas. Evidence. Impact.
The Population Council confronts critical health and development issues-from stopping the spread of HIV to improving reproductive health and ensuring that young people lead full and productive lives. Through biomedical, social science and public health research in about 50 countries, the Council works with our partners to deliver solutions that lead to more effective policies, programs, and technologies to improve lives worldwide. Established in 1952 and headquartered in New York, the Council is a nongovernmental, nonprofit organization with an international board of trustees. popcouncil.org

Suggested citation: Population Council. 2017. "Policymakers' perceptions of oral pre-exposure prophylaxis introduction in Tanzania: findings from implementation science research," DREAMS Tanzania Results Brief 6. Washington, DC: Population Council. 